ternus humeri sehnig und nur hoch oben auch von den sehnigen Theilen des E. digitorum communis und Ulnaris externus fleischig. Nachdem seine Selne die 5. Scheide des Lig. carpi dorsale passirt hat, theilt sie sich, wie oft in anderen gewöhnlichen Fällen, in zwei secundäre Sehnen, in eine schwächere radiale und in eine stärkere ulnare, welche sich am Rücken der Articulatio metacarpophalangea des kleinen Fingers vereinigen. Die secundäre radiale Sehne wird von der Sehne des Extensor digitorum cormunis zum 5. Finger, knapp über der Articulatio metacarpo-phalangea V, aufgenommen. Nach Vereinigung der Sehne des Extensor digitorum communis und der Sehne des $E$. digiti minimi an der genannten Articulation verhalten sie sich, wie in anderen Fällen. Die secundäre ulnare Sehne des E. digiti minimi bildet die ulnare Hälfte des Ansatzschenkels an die Mittelphalange und den ulnaren Ansatzschenkel an die Endphalange des kleinen Fingers, während die verschmolzenen anderen Sehnen die Bildung der radialen Hälfte des Ansatzschenkels an die Mittelphalange und die des radialen Ansatzschenkels an die Endphalange übernehmen.

Der neue Fall ist zu den 3 Fällen eigener Beobachtung (No. 2, 3 und 5) und zu Wood's und Macalister's zwei Fällen zu zählen, in welchen die Sehne für den Daumen durch die 3. Scheide des Lig. carpi dorsale mit der Sehne des Extensor pollicis longus trat; er hat einige Besonderheiten an sich; ist auch der erste, welcher beim weiblichen Geschlechte beobachtet worden ist.

\title{
X. (CXXVIII.) Nachtrag zum Vorkommen des Museulus tensor laminae posterioris vaginae musculi recti abdominis.
}

$$
\text { (3. Fall.) }
$$

Ich kenne diesen Muskel seit 1860 und habe davon bis jetzt 2 Fälle mitgetheilt ${ }^{1}$ ), abgesehen von dem von mir $^{2}$ ) und den von Tarenetzky ${ }^{3}$ ) veröffentlichten Făllen von "Tensor laminae posterioris vaginae musculi recti et fasciae transversae abdominis".

1) a) Ueber einige supernumeräre Bauchmuskeln. No. III. Bulletin de l'Acad. Imp. d. sc. de St. Petersbourg. Tom. XVIII. 1872. Col. 144. [An beiden Seiten eines Mädchens (1. Fall).] - b) Anat. Notizen No. LXXXIl. Díeses Archiv Bd. 69. Berlin 187\%. S. 400. [An der rechten Seite eines männlichen Individuums (2. Fall).]

2) Citirter Aufsatz: a. Daselbst Col. 144-147. Holzschnitt.

3) Topographische Beschreibung der Reg. hypogastrica propria. Dissertation (russisch). St. Petersburg 1874. S。 43. Fig. No. 6 a, b b. (Auszug im citirten Aufsatz, b.) 
Am 1. Februar $1879 \mathrm{kam}$ mir derselbe Muskel an der Leiche eines jungen Mannes beiderseitig wieder zur Beobachtang.

Gestalt und Grösse. Ein länglich-dreiseitiger, gegen seine Endigung allmählich an Breite abnehmender, dünner Muskel, von $10-11 \mathrm{Cm}$. Länge, wovon $9 \mathrm{Cm}$. auf die Fleischportion bommen; an dieser am Anfange $3 \mathrm{Cm}$, am Ende $6 \mathrm{Mm}$. breit und bis $2 \mathrm{Mm}$. dick.

Uraprung. Vom Os pubis hinter dem Rectus abdominis mit einem Sehnenbïndel, dann mit isolirten theils sogleicb fleischigen, theils im Anfange sehnigen Bündeln, in einer Strecke von $3 \mathrm{Cm}$. bis zu einer Distanz von $2,2 \mathrm{Cm}$. vom Annulus internus canalis inguinalis auswärts, von der unrichtig als Ligamentum Colles angeführten, äusseren oberen, im Lig. ilio-pubicum endenden Partie des Crus internum der Aponeurose des M. obliquus externus abdominis der entgegengesetzten Seite (Tarenetzky) und in dem Winkel, welcher durch die Verbindung der Fascia transversa mit der hinteren Kante des Arcus cruralis entsteht.

Verlauf. Ausserhalb der Vaginae mm. rectorum abdominis, neben diesen auf der Fascia transversa in eine Zellscheide eingehüllt, zuerst im Trigonum canalis inguinalis, hier den Funiculus spermaticus von hinten kreuzend, dann hinter dem M. transversus abdominis, die Vasa epigastrica von vorn her kreuzend, wenig schräg anf- und auswärts.

Endigung. Mit einer $1-2 \mathrm{~cm}$. langen Aponeurose mit divergirenden Fasern im Horn der Plica Douglasii des hinteren Blattes der Vagina rectorum und darüber in diesen.

\section{XI. (CXXIX.) Nachtrag zn den Beobachtungen über den Mangel des Musenlus quadratus femoris. \\ (12. Fall eigener Beobachtung.)}

Ich hatte bis jetzt den Musculus quadratus femoris an 11 Hüften von 8 Cadavern ( 7 männlichen und 1 weiblichen) vermisst, worüber ich bereits Mittheilung gemacht habe').

Am 28. October 1878 wurde der Muskel an der linken Extremität eines robusten Mannes wieder vermisst. Der Obturator externus, vom Nervus ischiadicus gekreuzt, lag frei zu Tage.

Mir ist somit Mangel des M. quadratus femoris an 12 Hüften von 9 Cadavern ( 8 männlichen und 1 weiblichen) vorgekommen und zwar nach der Zahl der Gadaver in $\frac{1}{3}$ beiderseitig und in $\frac{2}{3}$ einseitig; nach der Zahl der Hüften in $\frac{1}{3}$ rechtsseitig und in $\frac{2}{3}$ linksseitig. Einseitiger und linksseitiger Mangel sind überwiegend oft vorgekommen.

1) Anatomische Notizen No. CVII. Dieses Archiv Bd. 73. 1878. S. 346. 